\title{
Effectiveness of a procalcitonin algorithm to guide antibiotic therapy in respiratory tract infections outside of study conditions: a post-study survey
}

\author{
P. Schuetz $\cdot$ M. Batschwaroff • F. Dusemund • \\ W. Albrich • U. Bürgi • M. Maurer • M. Brutsche • \\ A. R. Huber • B. Müller
}

Received: 6 September 2009 / Accepted: 26 November 2009/Published online: 29 December 2009

(C) Springer-Verlag 2009

\begin{abstract}
All published evidence on procalcitonin (PCT)guided antibiotic therapy was obtained in trials where physicians knew that they were being monitored, possibly resulting in higher adherence to the PCT algorithm. This study investigates the effectiveness of PCT guidance in an observational quality control survey. We monitored antibiotic therapy and algorithm adherence in consecutive patients with respiratory tract infections admitted to the Kantonsspital Aarau, Switzerland, between May 2008 and February 2009. The results were compared to the sitespecific results of the former ProHOSP study. Overall and more pronounced for patients with community-acquired pneumonia, the median duration of antibiotic treatment in this survey was shorter than the ProHOSP control patients ( 6 vs. 7 days, $P=0.048$ and 7 vs. 9 days, $P<0.001$ ). In $72.5 \%$ of patients, antibiotics were administered according to the prespecified PCT algorithm. No significant differ-
\end{abstract}

P. Schuetz, M. Batschwaroff and F. Dusemund are equally contributing first authors.

\section{P. Schuetz}

Division of Internal Medicine, University Hospital Basel, 4031 Basel, Switzerland

P. Schuetz $\cdot$ M. Batschwaroff $\cdot$ F. Dusemund $\cdot$ W. Albrich $\cdot$

U. Bürgi $\cdot$ M. Maurer $\cdot$ M. Brutsche $\cdot$ B. Müller $(\varangle)$

Department of Internal Medicine and Division of Pulmology,

Kantonsspital Aarau,

Tellstrasse,

5001 Aarau, Switzerland

e-mail: happy.mueller@unibas.ch

\section{A. R. Huber}

Department of Laboratory Medicine,

Kantonsspital Aarau,

Tellstrasse,

5001 Aarau, Switzerland ences concerning adverse medical outcome could be detected. This study mirrors the use of PCT-guided antibiotic therapy in clinical practice, outside of trial conditions. If algorithm adherence is reinforced, antibiotic exposure can be markedly reduced with subsequent reduction of antibiotic-associated side effects and antibiotic resistance. The integration of the PCT algorithm into daily practice requires ongoing reinforcement and involves a learning process of the prescribing physicians.

\section{Introduction}

In the last five years, seven consecutive randomised controlled trials (RCTs) have demonstrated the efficacy, effectiveness, feasibility and safety of procalcitonin (PCT)guided antibiotic stewardship in patients with lower respiratory tract infections (LRTI) and sepsis [1-7]. Thereby, embedded in an easy-to-use and pragmatic clinical algorithm, the initiation or continuation of antibiotics was more or less discouraged or encouraged, respectively, based on circulating PCT levels (Fig. 1). To assure practicability and safety for patients, specific 'overruling' criteria were predefined, where this algorithm could be bypassed (e.g. imminent life-threatening disease or the need for intensive care unit [ICU] admission) [8]. Overall, PCT-guided antibiotic stewardship reduced the initial antibiotic prescription rate by $40-50 \%$ in patients with LRTI presenting to the emergency room [5], by $72 \%$ in ambulatory patients presenting for an outpatient visit with the general physician [13] and total antibiotic exposure in patients with community-acquired pneumonia (CAP) by $48 \%$ [4], mainly by the shortening of antibiotic courses without increasing the rate of adverse outcomes [1-7]. 


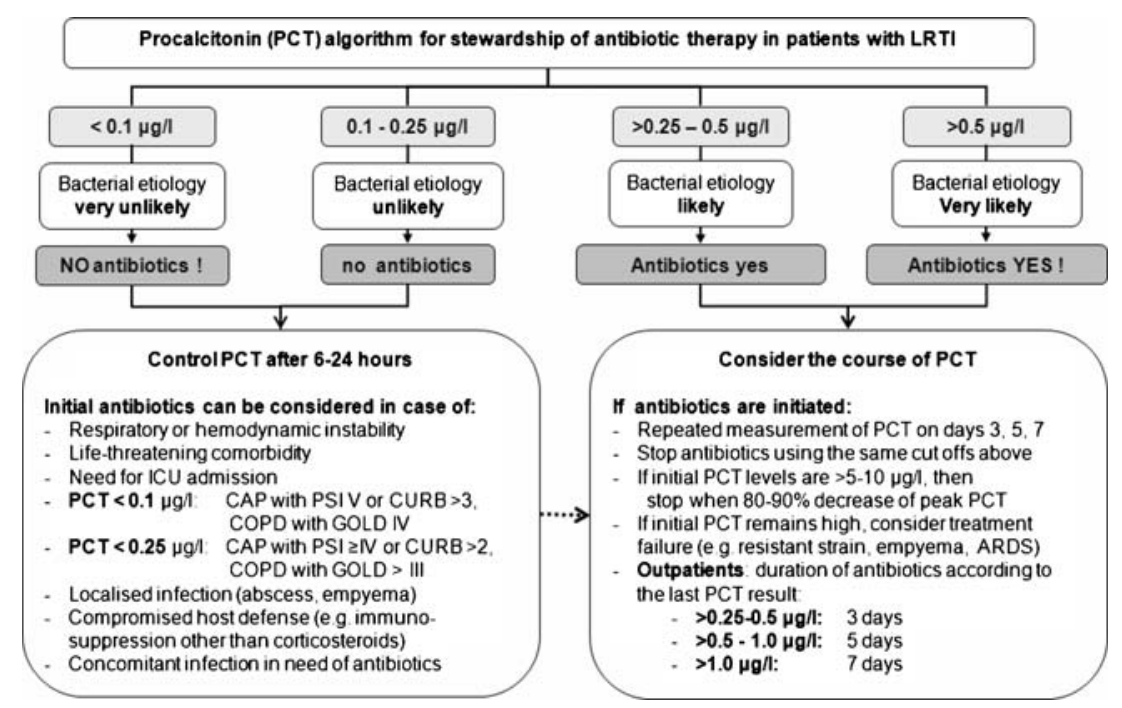

Fig. 1 Procalcitonin (PCT) algorithm

All published evidence on PCT-guided antibiotic stewardship was obtained from RCTs, and data on the effectiveness outside of controlled study conditions are lacking. Results from RCTs may not unconditionally be applied to general patients due to exclusion criteria or nonenrollment, limiting generalisability. In addition, it is well known that results obtained in RCTs are frequently not adequately implemented in daily practice. For instance, patients with CAP are often admitted to in-hospital care, despite their low risk for mortality as assessed with the pneumonia severity index (PSI) and guideline recommendation to treat as outpatients $[9,10]$. Non-adherence to CAP guidelines in real life can also be observed in relation to other decisions, e.g. the timely administration of antibiotics or collection of blood cultures. An impressive example of an unanticipated effect of introducing a study result was a marked increase in the rates of hyperkalaemia observed in the post-study population-based surveillance in patients with congestive heart failure treated with spironolactone according to the results of the RALES trial [11]. Of note, the risk of hyperkalaemia was listed as rare in the original RALES report [12].

In this context, this survey aims to investigate the effectiveness of PCT-guided antibiotic stewardship in an observational quality control survey to better mirror the 'real-life' setting and, thereby, to prevent misuse.

\section{Methods}

Subjects and study design

This quality control survey monitored the initiation and duration of antibiotic therapy, adherence to the published
PCT algorithm (Fig. 1) and outcome of all consecutive patients with LRTI admitted to the Kantonsspital Aarau, Switzerland, between May 2008 and February 2009. The same hospital participated until March 2008 as an active recruitment site in the multicentre ProHOSP study [2, 7]. This study prospectively randomised patients to receive antibiotic therapy based either on PCT cut-off ranges (PCT intervention group) or based on enforced guidelines (control group). For this survey, the diagnostic work-up, including chest radiograph and the treatment of patients, was left to the discretion of the treating physicians. PCT was measured in all patients with LRTI in a clinical routine using a highly sensitive immunoassay with a functional assay sensitivity of $0.06 \mu \mathrm{g} / \mathrm{L}$ (Kryptor ${ }^{\circledR}$, BRAHMS AG, Hennigsdorf, Germany).

For this survey, all patients with LRTI who were admitted to the hospital were Web-based registered by the physician on duty. Physicians were blinded to the aim of the survey and reminded of the need for registration threemonthly. The same PCT algorithm which was used during the ProHOSP study (Fig. 1) was displayed in the emergency department. New resident and attending physicians were briefed about this surveillance study.

The local Institutional Review Board (Kantonale Ethikkommission Aargau, Departement Gesundheit und Soziales) classified this study as an observational quality surveillance and, thus, waived the need for patient informed consent.

\section{Definitions}

Similar to the definition used in the ProHOSP study, LRTI was defined as the presence of at least one respiratory symptom (cough, sputum production, dyspnoea, tachyp- 
noea, pleuritic pain) plus at least one finding during auscultation (rales, crepitation) or one sign of infection (core body temperature $>38.0^{\circ} \mathrm{C}$, shivering, leukocyte count $>10 \mathrm{G} / 1$ or $<4 \mathrm{G} / 1$ cells) independent of antibiotic pretreatment. In accordance with guidelines, CAP was defined as a new infiltrate on chest radiograph [13-16]. GOLD criteria were used to define chronic obstructive pulmonary disease (COPD) by post-bronchodilator spirometric criteria as an FEV1/FVC ratio below $70 \%$ and to categorise the severity $[13,17]$. Acute bronchitis was defined as LRTI in the absence of an underlying lung disease or focal chest signs and infiltrates on chest radiograph, respectively [14].

\section{Monitoring of patients}

Upon admission, the following data were prospectively recorded by a member of the study team: age, gender and comorbidity of patients, results of prognostic and diagnostic work-up, i.e. parameters included in the PSI [10] and CURB65 score, blood culture and urine antigen test for Legionella pneumophila. After discharge of the patients, the database was completed with information about the duration and route of antibiotic therapy, total length of hospital stay and adverse medical outcomes, including all-cause inhospital mortality, ICU admission, complications (e.g. empyema) and recurrence rate during the index hospitalisation. The survey ended when patients were discharged from the hospital and we did not perform additional follow up.

\section{Endpoints}

The primary endpoint of this study was the total duration of antibiotic treatment. We used data from the ProHOSP study participants of the same hospital to compare the results of this survey. Thereby, we used the ProHOSP intervention group as positive controls and the ProHOSP control group as negative controls.

Secondary endpoints were adherence to the PCT algorithm, adverse medical outcomes, all-cause mortality, ICU admission, complications and recurrence rate within the index hospitalisation and length of hospital stay. Adherence with the PCT algorithm was evaluated independently by two members of the research group (MB and PS) after a patient was discharged. The evaluation was based on adherence to the previously published PCT algorithm (Fig. 1). In brief, the initiation or continuation of antibiotics was discouraged if PCT levels were $\leq 0.25 \mu \mathrm{g} / \mathrm{L}$ and encouraged if PCT levels were $>0.25 \mu \mathrm{g} / \mathrm{L}$. In addition, in patients with very low PCT levels $(<0.1 \mu \mathrm{g} / \mathrm{L})$, antibiotics were strongly discouraged, while they were strongly encouraged in patients with PCT levels $>0.5 \mu \mathrm{g} / \mathrm{L}$. In case antibiotics were withheld, clinical re-evaluation and a repeated measurement of PCT was recommended after 6-
$24 \mathrm{~h}$. If PCT values were increased and antibiotic therapy was initiated, repeated PCT measurements were recommended and antibiotics were discontinued using the same cut-off ranges. In patients with very high initial PCT values (i.e. $>5-10 \mu \mathrm{g} / \mathrm{L}$ ), the discontinuation of antibiotic therapy was encouraged if levels decreased by $90 \%$ or at least $80 \%$ of the initial value. In addition, the same specific 'overruling' criteria were defined as in the initial studies [7] where this algorithm could be bypassed (e.g. lifethreatening disease or immediate need for ICU admission). Accordingly, non-adherence was defined if antibiotic therapy was initiated or not discontinued, despite low PCT levels in the absence of any of the predefined criteria.

\section{Statistical analysis}

Discrete variables were expressed as counts (percentage) and continuous variables as medians and interquartile ranges (IQR), unless stated otherwise. Frequency comparison was done by the Chi-square test. Two-group comparison of normally distributed data was performed by Student's t-test. For data not normally distributed, the Mann-Whitney $U$-test was used. All statistical analyses were done by the SPSS Statistics package (version 17.0, SPSS Inc., Chicago, IL, USA) and STATA 9.2 (Stata Corp., College Station, TX, USA). All testing was two-tailed and $P$-values less than 0.05 were considered to indicate statistical significance.

\section{Results}

Baseline characteristics

Three hundred and two patients were included in this survey with a median age of 71 years and $68 \%$ were male. CAP was diagnosed in $71.9 \%$, while in $18.5 \%$ acute bronchitis and in $9.6 \%$ acute exacerbation of COPD was diagnosed. Table 1 shows the baseline characteristics of the survey population as compared to patients in the control and the intervention group of the ProHOSP study. Clinical findings and most co-existing illnesses at presentation were similar in all three of the compared groups. Neoplastic disease and immunosuppression were more frequent in the survey population. In addition, survey patients had a higher frequency of CAP with higher severity as assessed with the PSI. In addition, the initial serum levels of PCT, C-reactive protein and white blood cell count were similar in the survey population as compared to ProHOSP patients.

Primary endpoint: duration of antibiotic treatment

The overall median duration of antibiotic treatment in the survey population of 6 days was lower than in the 
Table 1 Baseline characteristics of patients included in the survey and the ProHOSP study

$\begin{array}{lllll}\text { Survey }(n=302) & \begin{array}{l}\text { ProHOSP control } \\ \text { group }(n=121)\end{array} & \begin{array}{l}\text { ProHOSP } \\ \text { intervention } \\ \text { group }(n=116)\end{array} & \begin{array}{l}\text { Comparison: } \\ \text { survey vs. } \\ \text { control group }\end{array} & \begin{array}{l}\text { Comparison: } \\ \text { survey vs. } \\ \text { intervention group }\end{array}\end{array}$

Comparison: survey vs.

Demographic characteristics

Age (years)

Sex (male), no. (\%)

Coexisting illnesses, no. (\%)

Coronary heart disease

Cerebrovascular disease

$71(55-81)$

$186(68 \%)$

$67(49-80)$

$72(59.5 \%)$

$44(14.6 \%)$

$0(0 \%)$

$118(39.1 \%)$

$112(37.1 \%)$

$85(28.1 \%)$

$20(6.6 \%)$

$56(18.5 \%)$

Diabetes

Clinical findings

Confusion, no. (\%)

Respiratory rate (breaths/min)

Systolic blood pressure (mmHg)

Heart rate (beats/min)

Body temperature $\left({ }^{\circ} \mathrm{C}\right)$

Laboratory findings

Procalcitonin $(\mu \mathrm{g} / \mathrm{l})$

C-reactive protein $(\mathrm{mg} / \mathrm{l})$

WBC $\left(\times 10^{9} / 1\right)$

Final diagnosis, no. (\%)

CAP

Exacerbation of COPD

Bronchitis

Other final diagnosis

Risk assessment in CAP patients

PSI points

PSI class IV or V, no. (\%)

CURB65

$\begin{array}{ll}30(9.9 \%) & 4(3.3 \%) \\ 24(20-28) & 20(16-25) \\ 130(114-145) & 125(120-140) \\ 97(80-110) & 92(80-105) \\ 37.9(37-38.6) & 37.9(37.2-38.8) \\ & \\ 0.28(0.12-1.08) & 0.21(0.08-0.93) \\ 90(28-185) & 87(29-172) \\ 10.9(7.5-15.2) & 10.9(8-14.5) \\ & \\ 217(71.9 \%) & 66(54.5 \%) \\ 29(9.6 \%) & 25(20.7 \%) \\ 56(18.5 \%) & 26(21.5 \%) \\ - & 4(3.3 \%) \\ 103(72-129) & 84(52-106) \\ 185(61.3 \%) & 53(43.8 \%) \\ 1(1-2) & 1(0-2)\end{array}$

$4(3.3 \%)$

(16-25)

$92(80-105)$
$21(17.4 \%)$

$8(6.6 \%)$

$29(24 \%)$

$48(39.7 \%)$

$14(11.6 \%)$

$1(0.8 \%)$

$22(18.2 \%)$

Data are expressed as median (interquartile range, IQR), unless stated otherwise

ProHOSP control group ( 7 days, $P<0.05$ ), and tended to be higher compared to the ProHOSP intervention group (4 days, $P=0.08$ ). In the subgroup of patients with CAP, the median duration of antibiotic treatment in the survey was significantly lower compared to the ProHOSP control group ( 7 days vs. 9 days, $P<0.001$ ) and similar to the ProHOSP intervention group ( 7 days vs. 7 days, $P=0.24$ ). Detailed antibiotic courses of CAP patients in the different study groups are presented in Fig. 2. In the subgroup of patients with exacerbation of COPD, the overall treatment duration was again lower in the survey as compared to the ProHOSP control group ( 0 days vs. 4 days, $P<0.05)$ and tended to be lower as compared to the intervention group (0 days vs. 3 days, $P=0.08$ ). For patients with bronchitis, no significant

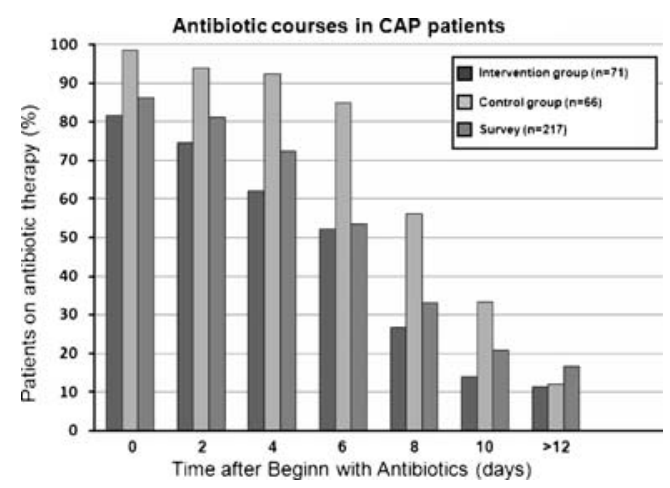

Fig. 2 Course of antibiotic treatment in community-acquired pneumonia (CAP) patients from the ProHOSP (intervention and control group) study and survey patients 
Table 2 Antibiotic prescription and outcome in patients included in the survey and the ProHOSP study

\begin{tabular}{|c|c|c|c|c|c|}
\hline & $\begin{array}{l}\text { Survey } \\
\text { population }\end{array}$ & $\begin{array}{l}\text { ProHOSP } \\
\text { control group }\end{array}$ & $\begin{array}{l}\text { Comparison: } \\
\text { survey vs. } \\
\text { control group }\end{array}$ & $\begin{array}{l}\text { ProHOSP } \\
\text { PCT group }\end{array}$ & $\begin{array}{l}\text { Comparison: } \\
\text { survey vs. } \\
\text { intervention group }\end{array}$ \\
\hline All patients & $(n=302)$ & $(n=121)$ & & $(n=116)$ & \\
\hline \multicolumn{6}{|l|}{ Duration of antibiotic treatment (days) } \\
\hline Median (quartiles) & $6(0-9)$ & $7(0-10)$ & $P=0.048$ & $4(0-8)$ & $P=0.08$ \\
\hline Mean & 5.99 & 7.1 & & 5 & \\
\hline Antibiotic prescription, $\%$ & 71.5 & 74.4 & $P=0.63$ & 63.8 & $P=0.16$ \\
\hline \multicolumn{6}{|l|}{ Adverse medical outcome } \\
\hline Overall adverse outcome, $\%$ & 21.5 & 24 & $P=0.61$ & 16.4 & $P=0.28$ \\
\hline Mortality, \% & 9.9 & 6.6 & $P=0.35$ & 7.8 & $P=0.58$ \\
\hline ICU admission, \% & 7 & 13.2 & $P=0.06$ & 7.8 & $P=0.83$ \\
\hline Recurrence, \% & 3.3 & 5 & $P=0.42$ & 2.6 & $P=0.7$ \\
\hline Disease-specific complication, $\%$ & 3 & 3.3 & $P=1.0$ & 0.9 & $P=0.3$ \\
\hline Length of hospital stay, median (quartiles) & $8(5-13)$ & $6(2-11)$ & $P<0.001$ & $7(3-11)$ & $P=0.003$ \\
\hline CAP & $(n=217,71.9 \%)$ & $(n=66,54.6 \%)$ & & $(n=71,61.2 \%)$ & \\
\hline \multicolumn{6}{|l|}{ Duration of antibiotic treatment (days) } \\
\hline Median (quartiles) & $7(4-10)$ & $9(7-11)$ & $P<0.001$ & $7(2-9)$ & $P=0.24$ \\
\hline Mean & 7.5 & 9.39 & & 6.73 & \\
\hline Antibiotic prescription, $\%$ & 86.2 & 98.5 & $P=0.003$ & 81.7 & $P=0.35$ \\
\hline Overall adverse outcome, $\%$ & 25.3 & 31.8 & $P=0.34$ & 14.1 & $P=0.05$ \\
\hline Mortality, \% & 11.5 & 9.1 & $P=0.66$ & 7 & $P=0.37$ \\
\hline Length of hospital stay, median (quartiles) & $9(6-13)$ & $7(3-12)$ & $P=0.04$ & $7(3-11)$ & $P=0.006$ \\
\hline Exacerbation of COPD & $(n=29,9.6 \%)$ & $(n=25,20.7 \%)$ & & $(n=20,17.2 \%)$ & \\
\hline \multicolumn{6}{|l|}{ Duration of antibiotic treatment (days) } \\
\hline Median (quartiles) & $0(0-4)$ & $4(0-8)$ & $P=0.04$ & $3(0-7)$ & $P=0.08$ \\
\hline Mean & 1.97 & 3.84 & & 3.7 & \\
\hline Antibiotic prescription, $\%$ & 31 & 64 & $P=0.02$ & 60 & $P=0.08$ \\
\hline Overall adverse outcome, $\%$ & 17.2 & 16 & $P=1.0$ & 25 & $P=0.72$ \\
\hline Mortality, \% & 10.3 & 8 & $P=1.0$ & 10 & $P=1.0$ \\
\hline Length of hospital stay, median (quartiles) & $7(4-14)$ & $5(3-9)$ & $P=0.1$ & $10(4-12)$ & $P=0.98$ \\
\hline Bronchitis & $(n=56,18.5 \%)$ & $(n=26,21.5 \%)$ & & $(n=21,18.1 \%)$ & \\
\hline \multicolumn{6}{|l|}{ Duration of antibiotic treatment (days) } \\
\hline Median (quartiles) & $0(0-4)$ & $0(0-0)$ & $P=0.24$ & $0(0-0)$ & $P=0.07$ \\
\hline Mean & 2.21 & 1.5 & & 0.71 & \\
\hline Antibiotic prescription, $\%$ & 35.7 & 19.2 & $P=0.2$ & 14.3 & $P=0.09$ \\
\hline Overall adverse outcome, $\%$ & 8.9 & 15.4 & $P=0.46$ & 9.5 & $P=1.0$ \\
\hline Mortality, \% & 3.6 & 0 & $P=1.0$ & 4.8 & $P=1.0$ \\
\hline Length of hospital stay, median (quartiles) & $5(1-10)$ & $0(0-5)$ & $P=0.004$ & $0(0-7)$ & $P=0.01$ \\
\hline
\end{tabular}

difference was found between groups. Table 2 shows more details on the primary and secondary endpoints.

Secondary endpoints

Adverse medical outcome and length of hospital stay

A total of 65 patients (21.5\%) experienced adverse medical outcomes during the hospital stay: 30 patients died during the hospital stay and, thus, the in-hospital mortality rate was $9.9 \%$. Of the 30 patients who died, 28 suffered from severe comorbidities, most frequently progressive malignant disease or liver cirrhosis, which subsequently lead to the limitation of therapeutic interventions to a comfort level. A total of 21 patients $(7 \%)$ were transferred to the ICU. The rates of overall adverse outcomes, mortality, ICU admission, recurrence and disease-specific complications were similar in the survey and the ProHOSP control patients (see 
Table 2). This was also true for subgroups of patients with CAP, exacerbation of COPD and acute bronchitis. The median length of hospital stay was significantly shorter in both ProHOSP groups as compared to the survey population. In the second half of the survey, the median length of stay was significantly shorter than in the first (7 vs. 9 days, $P=0.016$ ) and was similar to the ProHOSP groups.

\section{Adherence to the PCT algorithm}

In this survey, in 219 (73\%) patients, antibiotics were administered according to the prespecified PCT algorithm. In the $83(27 \%)$ patients who were overruled, the most important overruling reasons were severe immunosuppression $(22 \%)$, other infections in need of antibiotics $(17 \%)$ and anticipated complications (11\%). Additionally, 37\% of patients were overruled because of the clinical judgement of the treating physician without a prespecified reason (Fig. 3). Overruled patients tended to be younger (median age 65 [IQR 51-77] vs. 72 [IQR 57-82], $P=0.07$ ), tended to have higher rates of immunosuppression $(10.8 \%$ vs. $5.0 \%, P=0.07)$ and had higher rates of positive blood cultures or legionella antigen tests $(15.6 \%$ vs. $6.4 \%, P=$ 0.01 ). Overruling rate tended to decrease during the study from $31 \%$ in the first half of the study to $24 \%$ in the second study phase $(P=0.15)$.

\section{Comparison between overruled and not overruled survey patients}

The duration of antibiotic treatment in the non-overruled group (4 days) was significantly shorter than in the overruled group (11 days, $P<0.001)$ (Table 3). Antibiotics were prescribed in 60.7 and $100 \%$, respectively $(P<0.001)$. The overall antibiotic exposure in the adherent group was similar to the ProHOSP intervention group.

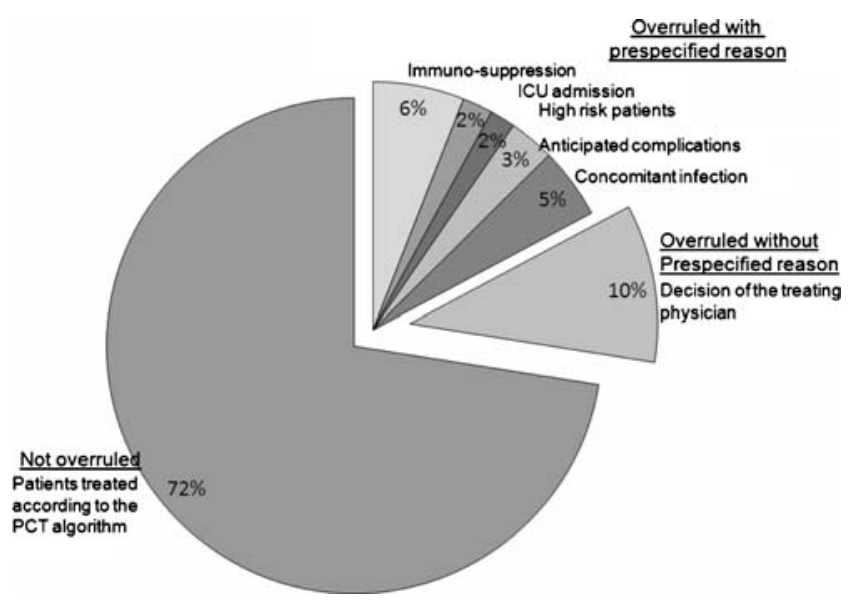

Fig. 3 Reasons for overruling

\section{Discussion}

In this observational survey, we found that in a real-life setting and outside of controlled study conditions, a previously validated PCT algorithm for antibiotic stewardship was effectively and safely implemented. In patients with immunosuppression, antibiotic therapy was not adapted according to the PCT algorithm, which complies with the lack of evidence in these patients, because immunosuppression was an exclusion criterion in all of today's published PCT intervention studies and these patients may be considered as high-risk patients.

In the randomised controlled, multicentre ProHOSP study, we previously showed that PCT-guided antibiotic stewardship markedly reduced antibiotic prescriptions in patients with LRTI presenting to the emergency room without increasing the risk of adverse outcomes [7]. Overall, the median duration of antibiotic treatment in the current survey population with LRTI was only moderately higher compared to the intervention group of the ProHOSP study and significantly lower as compared to the control group. For CAP patients, the survey population had similar rates compared to the intervention group and significantly lower rates as compared to the patients without PCT guidance in the control group. These results indicate that, also in a less stringent setting like in this survey, the use of $\mathrm{PCT}$ as a surrogate biomarker can shorten the period of antibiotic treatment.

Several of our observations merit further discussion. It is not entirely clear why the length of hospital stay in this current surveillance was longer than in the ProHOSP study groups treated at the same site within a 2-year time frame. The current survey included patients who were previously excluded, particularly immunocompromised patients or patients with severe comorbidities with expected imminent death or co-infections. This was mirrored in a higher proportion of patients with neoplastic disease in the survey population. However, these differences in LOS remained after the exclusion of these previously excluded subgroups from the analysis (data not shown). Alternatively, participation in the ProHOSP study might have created a Hawthorne effect influencing discharge behaviour. This seems less likely, as LOS was not the primary endpoint of the ProHOSP study and, furthermore, adverse outcomes were not different between the survey and the ProHOSP groups. Increasing use and confidence of physicians with PCT over time could lead to inappropriately relying on PCT as the sole or predominant marker influencing management decisions other than antibiotic use, thereby, jeopardising patients' safety. Importantly, this concern was not supported by the data of this survey as, e.g. ICU admission was similar in the survey and the ProHOSP intervention group. Interestingly, the length of hospital stay was significantly 
Table 3 Comparison of antibiotic prescription and outcome in relation to algorithm adherence in patients included in the survey; comparison with the ProHOSP control and PCT groups (values are presented in Table 2)

\begin{tabular}{|c|c|c|c|}
\hline & Non-overrulers & Overrulers & $\begin{array}{l}\text { Non-overrulers } \\
\text { vs. overrulers }\end{array}$ \\
\hline All patients & $(n=219)$ & $(n=83)$ & \\
\hline \multicolumn{4}{|l|}{ Duration of antibiotic treatment (days) } \\
\hline Median (quartiles) & $4(0-7)$ & $10(7-14)$ & $P<0.001$ \\
\hline Mean & 4.1 & 10.99 & \\
\hline Antibiotic prescription, $\%$ & 60.7 & 100 & $P<0.001$ \\
\hline \multicolumn{4}{|l|}{ Adverse medical outcome } \\
\hline Overall adverse outcome, $\%$ & 22.8 & 18.1 & $P=0.43$ \\
\hline Mortality, \% & 12.8 & 2.4 & $P=0.005$ \\
\hline ICU admission, $\%$ & 5.9 & 9.6 & $P=0.31$ \\
\hline Recurrence, \% & 4.1 & 1.2 & $P=0.21$ \\
\hline Disease-specific complication, $\%$ & 2.3 & 4.8 & $P=0.27$ \\
\hline Length of hospital stay, median (quartiles) & $8(5-12)$ & $9(6-15)$ & $P=0.02$ \\
\hline CAP & $(n=146)$ & $(n=71)$ & \\
\hline \multicolumn{4}{|l|}{ Duration of antibiotic treatment (days) } \\
\hline Median (quartiles) & $6(2-8)$ & $10(8-14)$ & $P<0.001$ \\
\hline Mean & 5.55 & 11.51 & \\
\hline Antibiotic prescription, $\%$ & 79.5 & 100 & $P<0.001$ \\
\hline Overall adverse outcome, $\%$ & 28.8 & 18.3 & $P=0.13$ \\
\hline Mortality, \% & 16.4 & 1.4 & $P<0.001$ \\
\hline ICU admission, \% & 6.8 & 11.3 & $P=0.27$ \\
\hline Length of hospital stay, median (quartiles) & $8(6-13)$ & $9(6-15)$ & $P=0.27$ \\
\hline Exacerbation of COPD & $(n=25)$ & $(n=4)$ & \\
\hline \multicolumn{4}{|l|}{ Duration of antibiotic treatment (days) } \\
\hline Median (quartiles) & $0(0-0)$ & $6(3-8)$ & $P=0.03$ \\
\hline Mean & 1.44 & 5.25 & \\
\hline Antibiotic prescription, $\%$ & 20 & 100 & $P=0.005$ \\
\hline Overall adverse outcome, $\%$ & 16 & 25 & $P=0.55$ \\
\hline Mortality, \% & 8 & 25 & $P=0.37$ \\
\hline ICU admission, \% & 8 & 0 & $P=0.56$ \\
\hline Length of hospital stay, median (quartiles) & $7(4-12)$ & $15(6-24)$ & $P=0.2$ \\
\hline Bronchitis & $(n=48)$ & $(n=8)$ & \\
\hline \multicolumn{4}{|l|}{ Duration of antibiotic treatment (days) } \\
\hline Median (quartiles) & $0(0-2)$ & $8(4-15)$ & $P<0.001$ \\
\hline Mean & 1.04 & 9.25 & \\
\hline Antibiotic prescription, $\%$ & 25 & 100 & $P<0.001$ \\
\hline Overall adverse outcome, $\%$ & 8.3 & 12.5 & $P=0.55$ \\
\hline Mortality, \% & 4.2 & 0 & $P=1.0$ \\
\hline ICU admission, \% & 2.1 & 0 & $P=0.68$ \\
\hline Length of hospital stay, median (quartiles) & $5(0-10)$ & $7(4-14)$ & $P=0.29$ \\
\hline
\end{tabular}

shorter in the second period of the survey in parallel to the improved adherence to the PCT algorithm.

An important focus of this study was to evaluate the adherence to the PCT algorithm outside of stringent study conditions. In $72.5 \%$ of cases, patients were treated according to the algorithm. In $63 \%$ of overruled cases, predefined overruling criteria (e.g. respiratory or haemodynamic instability, need for ICU admission, severe immunosuppression or a concomitant infection in need of antibiotics) were respected, while in $37 \%$ of overruled patients, no obvious reason could be detected. Thus, we achieved an overall algorithm compliance of $90 \%$ outside 
of study conditions, which is remarkable. Of note, the adherence to the study algorithm was $91 \%$ and, thus, similar to the ProHOSP trial [7]. The increasing compliance with the algorithm over time likely resulted from a learning effect and increasing confidence in PCT as a marker of the treating physicians and becoming familiar with the PCTalgorithm per se during the course of the study.

Within the survey cohort of patients, patients in the overruled group had significantly more and longer antibiotic treatment courses than the adherent group. The overall antibiotic exposure in the survey cohort was similar to the ProHOSP intervention group. There was no difference in the overall adverse medical outcome between overrulers and non-overrulers, despite the markedly shorter period of antibiotic therapy. This again approves the safety of the use of the PCT algorithm and demonstrates the great potential of PCT-guided therapy to reduce antibiotic exposure safely.

Of note, overruling occurs predominantly in patients with low PCT levels below the respective cut-off ranges. Mortality was significantly higher in the not overruled group. This is explained by the fact that patients in this group had higher PCT levels on admission to the hospital, indicating a more severe bacterial infection and, thereby, a poorer prognosis $[4,11,12]$. On the other hand, patients in which overruling occurred had, by definition, lower PCT values with subsequently lower risk of bacterial infection and, rather, a higher chance of self-limiting viral aetiology with low mortality $[5,11,12]$.

PCT-guided antibiotic stewardship has great potential to reduce the antibiotic mis- and overuse, which has been repeatedly shown to be directly linked to bacterial resistance in many settings [15-17]. As the effect of PCT on antibiotic consumption other than in study conditions has been unknown, this surveillance is encouraging and provides important new insights and, thus, may further improve the medical management of patients. Our current results mirror the use of PCT-guided antibiotic therapy in clinical practice and outside of trial conditions and demonstrate the feasibility of excellent adherence to the algorithm in real life with the use of only minor reminders, such as the instruction of new employees and posted leaflets in work areas. Antibiotic exposure strongly depended on this adherence. Using these informal reinforcements, antibiotic exposure can be markedly reduced, with subsequent reduction of antibiotic-associated side effects, and possibly of antibiotic resistance, and health care costs.

The strengths of this investigator-initiated observational post-study survey are the investigation of a biomarker strategy to reduce antibiotic over-prescription in real life and the comparison of results with a well defined cohort of LRTI patients from a previous study at the same institution. Our study has limitations. First, a pre-post design may not account for changes in routine practice during the study time period and may, thus, overestimate the effect of intervention. Second, the comparison of primary and secondary endpoints between both cohorts is limited by differences in the two patient populations due to different inclusion criteria; while this survey included all patients with LRTI, the ProHOSP study excluded some high-risk patients, i.e. patients with immediate expected adverse outcomes and immunosuppression. We expect a higher complication rate and longer antibiotic courses in these patients and this bias should, therefore, be conservative. Third, as a former ProHOSP hospital, physicians in this single-centre study were used to the treatment algorithm, which potentially increases adherence. Thus, future studies should investigate the effects of PCT guidance in different hospitals and outside of Switzerland.

In conclusion, utilisation of the PCT algorithm was feasible and effective in clinical practice outside of study conditions. Our observations indicate that the implementation of this algorithm requires time and can be understood as a learning process, where physicians become familiar with the PCT algorithm step by step. Given the critical association between adherence and effectiveness, ongoing reinforcement will likely facilitate the success of this intervention and, thus, ensure patient safety and the rational use of antibiotics, minimising the imminent threat of antibiotic resistance with individual and societal consequences. Multicentre surveillance is the next logical step to assure the quality of more widespread application of the algorithm.

Acknowledgement We are grateful to all of the patients, physicians and nursing staff from the Kantonsspital Aarau who participated in this survey.

Competing interests No commercial sponsor had any involvement in the design and conduct of this study, namely, collection, management, analysis and interpretation of the data, or preparation, decision to submit, review or approval of the manuscript. PS, WA and BM received support from BRAHMS to attend meetings and fulfil speaking engagements. BM has served as a consultant. All other authors declare that the answers to the questions on the competing interest form are all 'No' and, therefore, have nothing to declare.

Contributors PS, MBa and $\mathrm{BM}$ had the idea, initiated the study and wrote the protocol. PS, MBa, FD, WA, UB, BB, MBr, AH and BM managed the trial and collected data. PS, FD and WA performed the statistical analyses and drafted the manuscript. All authors amended and commented on the manuscript and approved the final version.

\section{References}

1. Stolz D, Christ-Crain M, Bingisser R, Leuppi J, Miedinger D, Müller C, Huber P, Müller B, Tamm M (2007) Antibiotic treatment of exacerbations of COPD: a randomized, controlled trial comparing procalcitonin-guidance with standard therapy. Chest 131(1):9-19 
2. Schuetz P, Christ-Crain M, Wolbers M, Schild U, Thomann R, Falconnier C, Widmer I, Neidert S, Blum CA, Schönenberger R, Henzen C, Bregenzer T, Hoess C, Krause M, Bucher HC, Zimmerli W, Müller B (2007) Procalcitonin guided antibiotic therapy and hospitalization in patients with lower respiratory tract infections: a prospective, multicenter, randomized controlled trial. BMC Health Serv Res 7:102

3. Christ-Crain M, Stolz D, Bingisser R, Müller C, Miedinger D, Huber PR, Zimmerli W, Harbarth S, Tamm M, Müller B (2006) Procalcitonin guidance of antibiotic therapy in communityacquired pneumonia: a randomized trial. Am J Respir Crit Care Med 174(1):84-93

4. Christ-Crain M, Jaccard-Stolz D, Bingisser R, Gencay MM, Huber PR, Tamm M, Müller B (2004) Effect of procalcitoninguided treatment on antibiotic use and outcome in lower respiratory tract infections: cluster-randomised, single-blinded intervention trial. Lancet 363(9409):600-607

5. Briel M, Schuetz P, Müller B, Young J, Schild U, Nusbaumer C, Périat P, Bucher HC, Christ-Crain M (2008) Procalcitonin-guided antibiotic use vs a standard approach for acute respiratory tract infections in primary care. Arch Intern Med 168:2000-2007

6. Nobre V, Harbarth S, Graf JD, Rohner P, Pugin J (2008) Use of procalcitonin to shorten antibiotic treatment duration in septic patients: a randomized trial. Am J Respir Crit Care Med 177(5): 498-505

7. Schuetz P, Christ-Crain M, Thomann R, Falconnier C, Wolbers M, Widmer I, Neidert S, Fricker T, Blum CA, Schild U, Regez K, Schönenberger R, Henzen C, Bregenzer T, Hoess C, Krause M, Bucher HC, Zimmerli W, Müller B (2009) Effect of procalcitoninbased guidelines vs standard guidelines on antibiotic use in lower respiratory tract infections: the ProHOSP randomized controlled trial. JAMA 302(10):1059-1066

8. Schuetz P, Christ-Crain M, Müller B (2009) Procalcitonin and other biomarkers to improve assessment and antibiotic stewardship in infections - hope for hype? Swiss Med Wkly 139(23-24):318-326
9. Lee RW, Lindstrom ST (2007) A teaching hospital's experience applying the Pneumonia Severity Index and antibiotic guidelines in the management of community-acquired pneumonia. Respirology 12(5):754-758

10. Fine MJ, Auble TE, Yealy DM, Hanusa BH, Weissfeld LA, Singer DE, Coley CM, Marrie TJ, Kapoor WN (1997) A prediction rule to identify low-risk patients with community-acquired pneumonia. N Engl J Med 336(4):243-250

11. Juurlink DN, Mamdani MM, Lee DS, Kopp A, Austin PC, Laupacis A, Redelmeier DA (2004) Rates of hyperkalemia after publication of the Randomized Aldactone Evaluation Study. N Engl J Med 351(6):543-551

12. Pitt B, Zannad F, Remme WJ, Cody R, Castaigne A, Perez A, Palensky J, Wittes J (1999) The effect of spironolactone on morbidity and mortality in patients with severe heart failure. Randomized Aldactone Evaluation Study Investigators. N Engl J Med 341(10):709-717

13. Calverley PM, Walker P (2003) Chronic obstructive pulmonary disease. Lancet 362(9389):1053-1061

14. Gonzales R, Sande MA (2000) Uncomplicated acute bronchitis. Ann Intern Med 133(12):981-991

15. Niederman MS, Mandell LA, Anzueto A, Bass JB, Broughton WA, Campbell GD, Dean N, File T, Fine MJ, Gross PA, Martinez F, Marrie TJ, Plouffe JF, Ramirez J, Sarosi GA, Torres A, Wilson R, Yu VL (2001) Guidelines for the management of adults with community-acquired pneumonia. Diagnosis, assessment of severity, antimicrobial therapy, and prevention. Am J Respir Crit Care Med 163(7):1730-1754

16. Woodhead M, Blasi F, Ewig S, Huchon G, Ieven M, Ortqvist A, Schaberg T, Torres A, van der Heijden G, Verheij TJ (2005) Guidelines for the management of adult lower respiratory tract infections. Eur Respir J 26(6):1138-1180

17. Anthonisen NR, Manfreda J, Warren CP, Hershfield ES, Harding GK, Nelson NA (1987) Antibiotic therapy in exacerbations of chronic obstructive pulmonary disease. Ann Intern Med 106(2):196-204 